\title{
Phylogenetic Analysis and Assessment of the Genera Vibrio, Photobacterium, Aeromonas, and Plesiomonas Deduced from Small-Subunit rRNA Sequences
}

\author{
RAYMOND RUIMY, ${ }^{1}$ VIOLETTE BREITTMAYER,${ }^{2}$ PHILIPPE ELBAZE, ${ }^{1}$ BÉNÉDICTE LAFAY, ${ }^{1}$ \\ ODILE BOUSSEMART, ${ }^{1}$ MICHEL GAUTHIER, ${ }^{2}$ AND RICHARD CHRISTEN ${ }^{1 *}$ \\ Centre National de la Recherche Scientifique and Université Paris 6, Station Zoologique, Villefranche-sur-Mer 06230, ${ }^{1}$ \\ and U303 Institut National de la Santé et de la Recherche Médicale, Nice 06000, ${ }^{2}$ France
}

\begin{abstract}
We sequenced nearly complete small-subunit rRNAs of 54 reference strains belonging to the genera Vibrio, Photobacterium, Aeromonas, and Plesiomonas. We then performed a phylogenetic analysis by comparing the sequences which we obtained with all other known sequences for bacteria belonging to the gamma subgroup of the Proteobacteria (thus providing a data base consisting of 70 sequences for the genera investigated), using methods such as neighbor joining, maximum likelihood, and maximum parsimony, as well as bootstrap, to assess the robustness of each topology. Our results confirmed that the family Vibrionaceae should include only Photobacterium and Vibrio species (but not Vibrio marinus); that Aeromonas species deserve family rank; and that Plesiomonas shigelloides is linked to the family Enterobacteriaceae. The genera Vibrio, Photobacterium, Aeromonas, and Plesiomonas, together with the family Enterobacteriaceae, the family Pasteurellaceae, and probably the genus Alteromonas, form a robust monophyletic unit within the gamma 3 subgroup of the Proteobacteria.
\end{abstract}

rRNA oligomer cataloging $(18,37,43)$, DNA-DNA and RNA-DNA hybridization $(3,6,18,32)$, and 5 S rRNA sequencing $(15,27,28)$ have been used previously to study the genera Vibrio, Photobacterium, Aeromonas, and Plesiomonas. Smallsubunit rRNA oligomer catalogs have revealed that these genera, along with the family Enterobacteriaceae, form a branch of the gamma 3 subdivision of the purple bacteria (43). More recently, inter- and intrageneric relationships have been determined by using small-subunit rRNA sequences of Vibrio strains (nearly complete sequences for 10 species [14] and partial sequences for Vibrio and Photobacterium species [23]) and Aeromonas strains (10 species), as well as Plesiomonas shigelloides (29). From a taxonomic point of view, broad general agreement has been reached between classification based on a molecular approach and classical classification derived from physiological comparisons and numerical taxonomy. The results of genetic studies suggested, however, that the family Vibrionaceae should include only the genera Vibrio and Photobacterium $(9,16,41,42)$, that the genus Aeromonas should be placed in a different family, the Aeromonadaceae, and that Plesiomonas shigelloides is more closely related to the family Enterobacteriaceae than to the family Vibrionaceae (6, $12,18,27,43)$.

Comparisons of small-subunit rRNA sequences are more informative than either analyses of 5S rRNA sequences or analyses of small-subunit rRNA oligomer catalogs. However, the results of studies in which workers used small-subunit rRNA sequences, which for many species were either not determined or were only partially determined, have left some questions unanswered. Also, the phylogenetic methods used previously to analyze relationships often have not included any estimate of the robustness of the results. Under these conditions, the phylogenetic relationships of all of these species were uncertain. In this study, we sequenced 54 strains and combined our data with previously published sequence data; thus, in our study we included data for almost all of the type strains of

* Corresponding author. Phone: (33) 937637 80. Fax: (33) 937638 93. Electronic mail address: christen@ccrv.obs-vlfr.fr. species in the genera Vibrio, Photobacterium, Aeromonas, and Plesiomonas. The nearly complete small-subunit rRNA sequences were aligned by comparison with other eubacterial rRNA sequences, and phylogenetic relationships were determined by using different phylogenetic methods (maximum likelihood, parsimony, neighbor joining) to check the reliability of each topology. Each topology was then analyzed by bootstrap to assess robustness.

\section{MATERIALS AND METHODS}

Bacterial strains. The bacterial strains belonging to the family Vibrionaceae for which small-subunit rRNA sequences are now available are listed in Table 1 . Table 1 shows the strain designations, sources of strains, GenBank or EMBL accession numbers for small-subunit rRNA sequences, and methods used to sequence either small-subunit rRNAs or ribosomal DNAs (rDNAs) when the sequences were determined in our laboratory.

All of the bacterial strains were grown in liquid medium (100 $\mathrm{ml})$ for $15 \mathrm{~h}$ at $22^{\circ} \mathrm{C}\left( \pm 2^{\circ} \mathrm{C}\right)$. The culture medium used was the medium recommended in the American Type Culture Collection Catalogue of Bacteria and Bacteriophages (1).

While our work was in progress, small-subunit rRNA sequences were published for some species whose sequences we had not determined at that time $(14,23,29,30)$. After these sequences were incorporated into our data base, alignment, and phylogenetic analyses, we found some phylogenetic discrepancies that let us to assume that some of the newly published sequences contained a few errors (for example, differences were observed in otherwise conserved domains in vibrios for the sequences of Vibrio hollisae, Vibrio diazotrophicus, Vibrio vulnificus, Vibrio proteolyticus, Vibrio alginolyticus, Vibrio parahaemolyticus, Vibrio campbellii, and Vibrio natriegens). For confirmation, some sequences (in particular, the sequences of $V$. alginolyticus, $V$. parahaemolyticus, and $V$. vulnificus) were determined both by reverse transcription directly from the rRNAs and by PCR from the genes (direct sequencing of PCR products [see below]), and no differences between the results of the two methods were observed. Also, 
TABLE 1. Strains belonging to the Vibrionaceae for which complete or nearly complete small-subunit rRNA sequences are now available

\begin{tabular}{|c|c|c|c|c|c|}
\hline \multirow{2}{*}{ Taxon } & \multirow{2}{*}{ Source(s) (strain[s] used $)^{a}$} & \multirow{2}{*}{ ATCC no. ${ }^{a}$} & \multicolumn{2}{|c|}{ Method $^{b}$} & \multirow{2}{*}{$\begin{array}{c}\text { EMBL } \\
\text { accession no. }\end{array}$} \\
\hline & & & rRNA & rDNA & \\
\hline Aeromonas allosaccharophila & $($ CECT 4199) & & & & \\
\hline Aeromonas caviae & CIP $\left(\text { CIP } 76.16^{\mathrm{T}}\right)^{d}$ & ATCC $15468^{\mathrm{T}}$ & ++ & & X74674 \\
\hline Aeromonas caviae & NCIMB (NCIMB 13016²) & & & & $\mathrm{X} 60408^{e}$ \\
\hline Aeromonas caviae & ATCC & ATCC 15467 & & & $\mathrm{X} 60409^{e}$ \\
\hline Aeromonas eucrenophila & $\operatorname{CIP}\left(\operatorname{CIP} 76.17^{\mathrm{T}}\right)$ & ATCC $23309^{\mathrm{T}}$ & ++ & & $\times 74675$ \\
\hline Aeromonas eucrenophila & NCIMB (NCIMB 74 ) & & & & $\mathrm{X} 60411^{e}$ \\
\hline Aeromonas hydrophila & CIP $\left(\right.$ CIP $\left.76.14^{\mathrm{T}}\right)$ & ATCC $7966^{\mathrm{T}}$ & ++ & & $X 74676$ \\
\hline Aeromonas hydrophila & NCIMB (NCIMB 9240 $)$ & ATCC $7966^{\mathrm{T}}$ & & & $\mathrm{X} 60404^{e}$ \\
\hline Aeromonas hydrophila & bioM & ATCC 35654 & ++ & & X74677 \\
\hline Aeromonas jandaei & ATCC & ATCC $49568^{\mathrm{T}}$ & ++ & & X74678 \\
\hline Aeromonas jandaei & ATCC & ATCC $49568^{\mathrm{T}}$ & & & $\mathrm{X} 60413^{e}$ \\
\hline Aeromonas media & bioM & ATCC $33907^{\mathrm{T}}$ & ++ & & X74679 \\
\hline Aeromonas media & ATCC & ATCC $33907^{\mathrm{T}}$ & & & $\mathrm{X} 60410^{e}$ \\
\hline Aeromonas salmonicida subsp. achromogenes & NCIMB (NCIMB $1110^{\mathrm{T}}$ ) & & & & $\mathrm{X} 60405^{e}$ \\
\hline Aeromonas salmonicida subsp. masoucida & $\mathrm{CIP}\left(\mathrm{CIP} 103210^{\mathrm{T}}\right)$ & ATCC $27013^{\mathrm{T}}$ & ++ & & $\mathrm{X} 74680$ \\
\hline Aeromonas salmonicida subsp. masoucida & & ATCC $27013^{\mathrm{T}}$ & & & $\mathrm{X} 60406^{e}$ \\
\hline Aeromonas salmonicida subsp. salmonicida & CIP $\left(\right.$ CIP $\left.103209^{T}\right)$ & ATCC $33658^{\mathrm{T}}$ & ++ & & X74681 \\
\hline Aeromonas salmonicida subsp. salmonicida & NCIMB (NCIMB1102 & & & & $\mathrm{X} 60407^{e}$ \\
\hline Aeromonas shubertii & bioM & ATCC $43700^{\mathrm{T}}$ & ++ & & $\times 74682$ \\
\hline Aeromonas shubertii & DSM (DSM 4882 $\left.{ }^{\mathrm{T}}\right)$ & & & & $\mathrm{X} 60416^{e}$ \\
\hline Aeromonas sobria & CIP (CIP $\left.74.33^{\mathrm{T}}\right)$ & ATCC $43979^{\mathrm{T}}$ & ++ & & X74683 \\
\hline Aeromonas sobria & CIP (CIP $\left.74.33^{\mathrm{T}}\right)$ & $\operatorname{ATCC} 43979^{\mathrm{T}}$ & & & $\mathrm{X} 60412^{e}$ \\
\hline Aeromonas sp. & & ATCC 35941 & & & $\mathrm{X} 60417^{e}$ \\
\hline Aeromonas trota & & ATCC $49657^{\mathrm{T}}$ & & & $\mathrm{X} 60415^{e}$ \\
\hline Aeromonas veronii & biom & ATCC $35624^{\mathrm{T}}$ & ++ & & X74684 \\
\hline Aeromonas veronii & ATCC & ATCC $35624^{\mathrm{T}}$ & & & $\mathrm{X} 60414^{e}$ \\
\hline Photobacterium angustum & $\operatorname{CIP}\left(\operatorname{CIP} 75.10^{\mathrm{T}}\right)$ & ATCC $25915^{\mathrm{T}}$ & ++ & + & X74685 \\
\hline Photobacterium leiognathi & $\operatorname{CIP}\left(\operatorname{CIP} 66.5^{\mathrm{T}}\right)$ & ATCC $25521^{\mathrm{T}}$ & ++ & + & X74686 \\
\hline Photobacterium phosphoreum & ATCC & ATCC $11040^{\mathrm{T}}$ & & ++ & $\times 74687$ \\
\hline Plesiomonas shigelloides & CIP $\left(\right.$ CIP $\left.63.5^{\mathrm{T}}\right)$ & ATCC $14029^{\mathrm{T}}$ & ++ & & X74688 \\
\hline Plesiomonas shigelloides & NCIMB (NCIMB 9242T) & & & & $\mathrm{X} 60418^{e}$ \\
\hline Vibrio aestuarianus & CIP $\left(\right.$ CIP $\left.102971^{\mathrm{T}}\right)$ & ATCC $35048^{\mathrm{T}}$ & ++ & + & $\times 74689$ \\
\hline Vibrio alginolyticus & $\operatorname{CIP}\left(\right.$ CIP $\left.17749^{\mathrm{T}}\right)$ & ATCC $17749^{\mathrm{T}}$ & $+t$ & $+t$ & $\times 74690$ \\
\hline Vibrio alginolyticus & & ATCC $17749^{\mathrm{T}}$ & & & $\mathrm{X} 56576^{f}$ \\
\hline Vibrio alginolyticus & CIP (CIP 7065) & & ++ & ++ & X74691 \\
\hline Vibrio (Listonella) anguillarum & & ATCC $19264^{\mathrm{T}}$ & & & $\mathrm{X} 16895^{g}$ \\
\hline Vibrio campbellii & $\operatorname{CIP}\left(\operatorname{CIP} 75.01^{\mathrm{T}}\right)$ & ATCC $25920^{\mathrm{T}}$ & & ++ & $\times 74692$ \\
\hline Vibrio campbellii & & ATCC $25920^{\mathrm{T}}$ & & & $\mathrm{X} 56575^{f}$ \\
\hline Vibrio carchariae & ATCC & ATCC $35084^{\mathrm{T}}$ & & ++ & $\times 74693$ \\
\hline Vibrio cholerae serovar $\mathrm{O} 1$ & CIP $\left(\right.$ CIP $\left.62.13^{\mathrm{T}}\right)$ & ATCC $14035^{\mathrm{T}}$ & ++ & + & X74694 \\
\hline Vibrio cholerae biotype Eltor, type inaba & INSERM U303 & ATCC 14033 & ++ & + & X74695 \\
\hline Vibrio cholerae non O:1 Nanking & CIP (CIP 63.40) & ATCC 14730 & ++ & + & X74696 \\
\hline Vibrio cholerae non O:1 El Tor & CIP (CIP 63.41) & ATCC 14731 & ++ & + & X74697 \\
\hline Vibrio cincinnatiensis & ATCC & ATCC $35912^{\mathrm{T}}$ & & ++ & X74698 \\
\hline Vibrio costicola & NCIMB (NCIMB 701) & ATCC $33508^{\mathrm{T}}$ & $+t$ & + & X74699 \\
\hline Vibrio (Listonella) damsela & CIP $\left(\right.$ CIP $\left.102761^{\mathrm{T}}\right)$ & ATCC $33539^{\mathrm{T}}$ & ++ & + & X74700 \\
\hline Vibrio diazotrophicus & CIP (CIP $103191^{\mathrm{T}}$ ) & ATCC $33466^{\mathrm{T}}$ & & ++ & X74701 \\
\hline Vibrio diazotrophicus & & ATCC $33466^{\mathrm{T}}$ & & & $\mathrm{X} 56577^{f}$ \\
\hline Vibrio fisheri & NCIMB (NCIMB 1281) & ATCC $7744^{\mathrm{T}}$ & ++ & + & $\times 74702$ \\
\hline Vibrio fluvialis & NCIMB (NCIMB 2249) & $\operatorname{ATCC} 33809^{\mathrm{T}}$ & ++ & + & X74703 \\
\hline Vibrio furnisii & CIP $\left(\right.$ CIP $\left.102972^{\mathrm{T}}\right)$ & ATCC $35016^{\mathrm{T}}$ & ++ & + & X74704 \\
\hline Vibrio gazogenes & CIP (CIP $103173^{\mathrm{T}}$ ) & ATCC $29988^{\mathrm{T}}$ & ++ & + & X74705 \\
\hline Vibrio harveyi & CIP (CIP $\left.103192^{\mathrm{T}}\right)$ & ATCC $14126^{\mathrm{T}}$ & & + & $\times 74706$ \\
\hline Vibrio harveyi & & ATCC $14126^{\mathrm{T}}$ & & & $\mathrm{X} 56578^{f}$ \\
\hline Vibrio hollisae & CIP (CIP 101886 $\left.{ }^{\mathrm{T}}\right)$ & ATCC $33564^{\mathrm{T}}$ & & ++ & $\times 74707$ \\
\hline Vibrio hollisae & & ATCC $33564^{\mathrm{T}}$ & & & $\mathrm{X} 56583^{f}$ \\
\hline Vibrio logei & CIP (CIP $\left.103204^{\mathrm{T}}\right)$ & ATCC 15382 & ++ & + & $\times 74708$ \\
\hline Vibrio marinus & CIP (CIP $\left.102861^{\mathrm{T}}\right)$ & ATCC $15381^{\mathrm{T}}$ & & ++ & X74709 \\
\hline Vibrio mediterranei & CIP (CIP 103203 ${ }^{\mathrm{T}}$ ) & & ++ & & X74710 \\
\hline Vibrio metschnikovii & bioM (NCTC 11170) & & ++ & + & X74711 \\
\hline Vibrio metschnikovii & CIP $\left(\right.$ CIP $\left.69.14^{\mathrm{T}}\right)$ & & ++ & + & X74712 \\
\hline Vibrio mimicus & CIP (CIP $\left.101888^{\mathrm{T}}\right)$ & ATCC $33653^{\mathrm{T}}$ & ++ & + & X74713 \\
\hline Vibrio natriegens & CIP (CIP $\left.103193^{\mathrm{T}}\right)$ & ATCC $14048^{\mathrm{T}}$ & & ++ & X74714 \\
\hline Vibrio natriegens & & ATCC $14048^{\mathrm{T}}$ & & & $\mathrm{X} 56581^{f}$ \\
\hline Vibrio navarrensis & $\operatorname{CIP}\left(\operatorname{CIP} 103381^{\mathrm{T}}\right)$ & & ++ & + & X74715 \\
\hline Vibrio nereis & CIP (CIP $103194^{\mathrm{T}}$ ) & ATCC $25917^{\mathrm{T}}$ & ++ & + & $\times 74716$ \\
\hline
\end{tabular}


TABLE 1-Continued

\begin{tabular}{|c|c|c|c|c|c|}
\hline \multirow{2}{*}{ Taxon } & \multirow{2}{*}{ Source(s) (strain[s] used) ${ }^{a}$} & \multirow{2}{*}{ ATCC no. ${ }^{a}$} & \multicolumn{2}{|c|}{ Method $^{b}$} & \multirow{2}{*}{$\begin{array}{c}\text { EMBL } \\
\text { accession no }\end{array}$} \\
\hline & & & rRNA & rDNA & \\
\hline Vibrio nigripulchritudo & CIP (CIP $\left.103195^{\mathrm{T}}\right)$ & ATCC $27043^{T}$ & ++ & + & X74717 \\
\hline Vibrio ordalii & CIP (CIP $103205^{\mathrm{T}}$ ) & ATCC $33509^{\mathrm{T}}$ & ++ & + & X74718 \\
\hline Vibrio orientalis & CIP (CIP $102891^{\mathrm{T}}$ ) & ATCC $33934^{\mathrm{T}}$ & ++ & + & X74719 \\
\hline Vibrio (Listonella) pelagius & CIP (CIP $102762^{\mathrm{T}}$ ) & ATCC $25916^{\mathrm{T}}$ & ++ & + & $\mathrm{X} 74720$ \\
\hline Vibrio parahaemolyticus & CIP (CIP 75.02 $)$ & ATCC $17802^{\mathrm{T}}$ & ++ & ++ & X74721 \\
\hline Vibrio parahaemolyticus & & ATCC $17802^{\mathrm{T}}$ & & & $\mathrm{X} 56580^{f}$ \\
\hline Vibrio parahaemolyticus & CIP (CIP 73.30) & & $+t$ & ++ & X74722 \\
\hline Vibrio proteolyticus & CIP (CIP $102892^{\mathrm{T}}$ ) & ATCC $15338^{\mathrm{T}}$ & & ++ & X74723 \\
\hline Vibrio proteolyticus & & ATCC $15338^{\mathrm{T}}$ & & & $\mathrm{X} 56579^{f}$ \\
\hline Vibrio splendidus & $\mathrm{CIP}\left(\mathrm{CIP} 102893^{\mathrm{T}}\right)$ & ATCC $33125^{\mathrm{T}}$ & $+t$ & + & X74724 \\
\hline Vibrio tubiashii & CIP (CIP $102760^{\mathrm{T}}$ ) & ATCC $19109^{T}$ & ++ & + & $X 74725$ \\
\hline Vibrio vulnificus & CIP (CIP $\left.75.04^{\mathrm{T}}\right)$ & ATCC $27562^{\mathrm{T}}$ & $+t$ & ++ & $X 74726$ \\
\hline Vibrio vulnificus & & ATCC $27562^{\mathrm{T}}$ & & & $\mathrm{X} 56582^{f}$ \\
\hline Vibrio vulnificus & bioM & ATCC 29307 & ++ & ++ & $X 74727$ \\
\hline
\end{tabular}

${ }^{a}$ The sources are the collections from which the bacteria were obtained: ATCC, American Type Culture Collection, Rockville, Md.; CIP, Collection Institut Pasteur, Paris, France; bioM, Institut bioMerieux, Marcy l'Etoile, France; NCTC, National Collection of Type Cultures, London, United Kingdom; NCIMB, National Collection of Industrial and Marine Bacteria, Aberdeen, United Kingdom.

${ }^{b}$ For sequences determined in our laboratory, the method used for sequencing is indicated. rRNA, sequence obtained directly from rRNA; rDNA, sequence obtained from amplification products; + , partially sequenced, ++ , nearly completely sequenced.

${ }^{c}$ Sequence obtained from reference 30.

${ }^{d} \mathrm{~T}=$ type strain.

${ }^{e}$ Sequence obtained from references 29 and 30.

${ }^{f}$ Sequence obtained from reference 14 .

${ }^{g}$ Sequence obtained from reference 40 .

no differences were observed for several strains of the same species (for example, $V$. alginolyticus and $V$. parahaemolyticus strains) (Table 1). Thus, because of the precautions which we took, we feel confident that our sequence data were more accurate than the data published previously.

Sequencing. We determined almost the entire small-subunit rRNA sequence (corresponding to positions 4 to 1425 in the Escherichia coli rRNA sequence for direct RNA sequences and to positions 29 to 1425 for PCR products) for each representative of the family Vibrionaceae. Vibrio anguillarum and some Aeromonas species were not sequenced because their sequences had been previously published $(29,30,40)$. For Vibrio harveyi, previously described partial sequence was completed (positions 88 to 118 in the $E$. coli sequence).

Direct small-subunit rRNA sequencing. Bacteria were pelleted by centrifugation for $10 \mathrm{~min}$ at $5,000 \mathrm{rpm}$. The pellet was treated with lysozyme $(2 \mathrm{mg} / \mathrm{ml})$ for $15 \mathrm{~min}$ at $37^{\circ} \mathrm{C}$ in a buffer containing $10 \mathrm{mM}$ Tris- $\mathrm{HCl}$ (pH 7.5), $1 \mathrm{mM}$ EDTA, and $20 \mathrm{mg}$ of sucrose per ml. After centrifugation for $10 \mathrm{~min}$ at $2,500 \mathrm{rpm}$, the resulting pellet was homogenized with an Ultra-turax homogenizer in $5 \mathrm{ml}$ of a solution containing $4 \mathrm{M}$ guanidinium thiocyanate, $50 \mathrm{mM}$ Tris- $\mathrm{HCl}(\mathrm{pH} 7.6), 4 \mathrm{mM}$ EDTA, and $2 \%$ $N$-lauryl-sarcosyl. Total RNA was separated from protein by phenol extraction; this procedure was repeated three times and was followed by two chloroform washes. The total RNA was ethanol precipitated, suspended in sterile distilled water to measure the ratio of $A_{260}$ to $A_{280}$, precipitated with sodium acetate and ethanol, and finally suspended in sterile water to a concentration of $2 \mu \mathrm{g} / \mu \mathrm{l}$. The quality of the RNA extract was determined by staining $1 \%$ agarose gels with ethidium bromide.

RNA sequencing was carried out by using the method of Sanger et al. (34), which was modified to accomodate reverse transcriptase in place of DNA polymerase (31) and was also modified as follows: DNA synthesis was carried out in two steps, a labeling step involving primer extension with limited concentrations of ${ }^{35} \mathrm{~S}-\mathrm{dATP}$, followed by a classical chain termination step in which dideoxynucleotides were used. The synthetic primers used were complementary to conserved eubacterial domains identified previously (25).

Small-subunit rDNA sequencing after PCR amplification. A pellet obtained after centrifugation for $10 \mathrm{~min}$ at 5,000 rpm was resuspended in $100 \mu \mathrm{l}$ of a lysis solution $(10 \mathrm{mM}$ Tris- $\mathrm{HCl}, 1$ mM EDTA, 1\% Triton X-100; pH 8), heated for $3 \mathrm{~min}$ at $100^{\circ} \mathrm{C}$, and then transferred on ice. After a single chloroform extraction, $8 \mu \mathrm{l}$ of supernatant was used in a PCR to amplify the small-subunit rRNA genes. The initial denaturation step consisted of heating the reaction mixture at $95^{\circ} \mathrm{C}$ for $180 \mathrm{~s}$, and this was followed by an annealing step $\left(52^{\circ} \mathrm{C}\right.$ for $\left.60 \mathrm{~s}\right)$ and an extension step $\left(72^{\circ} \mathrm{C}\right.$ for $\left.90 \mathrm{~s}\right)$. The thermal profile then consisted of 25 cycles of annealing at $52^{\circ} \mathrm{C}$ for $60 \mathrm{~s}$, extension at $72^{\circ} \mathrm{C}$ for $90 \mathrm{~s}$, and denaturation at $94^{\circ} \mathrm{C}$ for $30 \mathrm{~s}$. A final extension step was carried out at $72^{\circ} \mathrm{C}$ for $5 \mathrm{~min}$. The PCR products were analyzed on a $1 \%$ low-melting-point agarose gel that included a molecular weight standard for quantification of the PCR yield. DNA sequencing was performed by using a protocol described by Anderson et al. (2), with slight modifications. The amplification primers used gave a PCR product that was $1.5 \mathrm{~kb}$ long and corresponded to positions 8 to 1508 in the $E$. coli sequence. The primers used for sequencing were identical to those used for direct sequencing of small-subunit rRNAs.

Primer positions. Eleven DNA primers were used in the sequencing reactions. These primers corresponded to the following positions in the $E$. coli sequence: primer $\mathrm{S} 2$, positions 99 to 119 ; primer S3, positions 242 to 262 ; primer S4, positions 342 to 356 ; primer S6, positions 518 to 534; primer S8, positions 684 to 702 ; primer S10, positions 906 to 925 ; primer S12, positions 1099 to 1114; primer S14, positions 1223 to 1240 ; primer S15, positions 1384 to 1400 ; and primer S17, positions 1493 to 1509 . A reverse primer (positions 8 to 28 ) was used in conjunction with primer S17 to amplify the small-subunit rDNAs.

Phylogenetic analysis and alignment: general procedure. The phylogenetic data described below were obtained (i) by using successive alignment and phylogeny procedures, and (ii) 
by reinvestigating deep branching patterns after close relationships were determined.

In each phylogenetic analysis, we restricted the comparisons to nucleotide positions that were aligned without doubt. Some analyses were performed several times, with or without small domains that could have reached the point of saturation for mutations. Although this approach was probably not as efficient as carefully weighting each position independently, it was easier to use and was probably a reasonable compromise considering the possible problems of crossing-over that might have affected some of the sequences investigated (36).

We also performed some analyses several times by including and excluding particular species that had high rates of mutation or that were distant outgroups. Indeed, a model study in which rRNA sequences of echinoderms were used showed that the presence of distantly related outgroups brings random noise into an analysis and does not allow workers to resolve relationships among the more closely related species (35). Long branches for ingroups have the same effect as distant outgroups (that is, they bring noise into a phylogenetic analysis). Thus, monophyletic units containing such species are unstable because of highly variable sequences, and their presence can also affect neighboring taxa. In order to alleviate this problem, we performed phylogenetic analyses successively with all outgroups or with a set of outgroups restricted to the closest relatives, with or without ingroups that were characterized by long branches.

For each phylogenetic analysis, in order to keep computation time within reasonable limits, it was not possible to include all representatives of outgroups and ingroups in maximumparsimony and maximum-likelihood analyses. This problem was alleviated by performing multiple analyses with different outgroups and different ingroups (as determined from the neighbor-joining analysis). All sequence alignment and species selection operations were done by using computer programs developed by us and available on request from $\mathrm{R}$. Christen.

Phylogenetic methods. (i) Neighbor joining. A neighborjoining algorithm like that developed by Saitou and Nei (33) was used. The program was rewritten to include inputs and outputs compatible with the ribosomal data base and other programs developed in our laboratory (running on 386-compatible personal computers and above).

(ii) Maximum parsimony. The PAUP program (38) for MacIntosh computers was used. All topologies were first obtained by using the heuristic options. According to the time used, a branch and bound search was then undertaken by using the full data set or a restricted set of species selected on the basis of the results of the heuristic search. When several most-parsimonious trees were obtained, a $100 \%$ consensus tree was constructed and treated as the most-parsimonious tree for constructing figures. Finally, a bootstrap analysis was performed (heuristic option) to check each topology for robustness. We favored a large number of analyses with many different bacterial representatives and 100 replications for each analysis rather than a high number $(1,000)$ of bootstrap replications, since our experience showed that increasing the number of bootstrap replications above 100 usually had only a small influence on the results, compared with the choice of species.

(iii) Maximum likelihood. Routinely, the fDNAml program rewritten by G. J. Olsen (University of Illinois, Urbana) was used with a Hewlett-Packard model 700 workstation. All analyses were performed by using the global option (in fact, $\mathrm{F}$ Y G options), and each analysis was performed several times with species in a different order. For some topologies, the results were compared with the results of the DNAML pro- gram of Felsenstein (17). Interestingly, the two programs sometimes led to alternative, but very similar topologies, in particular when the results of maximum-likelihood, maximumparsimony, and neighbor-joining analyses were not in perfect agreement. Since these topologies were considered not resolved (intrageneric relationships within Vibrio species), we did not attempt to solve this problem in this study.

Finally, all trees were plotted by using a MacIntosh computer and a program developed by $M$. Gouy (University of Lyon, Villeurbanne, France) that allows transformation of a formal tree representation (Newick's format) into MacDraw drawings.

Only topologies that were found to be similar by all three methods were retained as "true trees." Recent theoretical works have indeed demonstrated that convergence of the results of all three methods is a very robust indication that the correct phylogeny has been determined $(20,22)$.

Domains used. Different parts of the small-subunit rRNA sequences were used for the different trees shown in this paper. For the tree shown in Fig. 1 we used positions 29 to 67,100 to 153,226 to 441,484 to 589,658 to 832,860 to 997,1035 to 1131 , 1138 to 1266 , and 1295 to 1357 ( $E$. coli numbering). For the tree shown in Fig. 2 we used positions 9 to 69,100 to 180,226 to 441,479 to 832,859 to 928,932 to 1004,1028 to 1132 , and 1137 to 1426 . For the tree shown in Fig. 3 we used positions 29 to 74,100 to 189,218 to 453,479 to 831,859 to 928,932 to 1004,1028 to 1132 , and 1137 to 1368 . To determine intrageneric relationships among Vibrio species (Table 2) we used positions 34 to 74,100 to 184 , and 185 to 1352 .

\section{RESULTS}

Phylogenetic positions of the genera Vibrio, Photobacterium, Aeromonas, and Plesiomonas within the Proteobacteria. A broad phylogenetic analysis clearly confirmed that all species belonging to the genera Vibrio, Photobacterium, Aeromonas, and Plesiomonas branch in the Proteobacteria subgroup of the Eubacteria (data not shown), a result in agreement with the results of all previous studies. The positions of these taxa within this major lineage are shown in Fig. 1, an unrooted tree that combines the results of a neighbor-joining analysis (shown in Fig. 1) with the topologies obtained by maximum-likelihood and parsimony analyses, as described in the legend to Fig. 1. This analysis revealed the major subgroups previously identified among the Proteobacteria (the alpha, beta, delta, gamma, and epsilon subgroups).

The genera Vibrio, Photobacterium, Aeromonas, and Plesiomonas clearly belonged to the gamma subgroup and were strongly associated with the Enterobacteriaceae, the Pasteurellaceae and related species, Alteromonas haloplanktis, and, to a lesser extent, Marinomonas vaga and some other bacteria. These taxa form a monophyletic branch within the gamma 3 subgroup defined previously (43). This result was always obtained by all three methods (maximum likelihood, neighbor joining, and maximum parsimony) and was observed even when different species were chosen as outgroups or ingroups (data not shown).

Plesiomonas shigelloides appeared to be closely related to the Enterobacteriaceae, while the genera Vibrio plus Photobacterium and the genus Aeromonas formed two well-separated taxa with deep roots. Other internal branches were less robust (the percentages obtained in a bootstrap analysis are indicated in Fig. 1). The phylogenetic relationships among these taxa were difficult to resolve in our analyses, in part because the topology of the internal branches changed according to which species were chosen as representatives of distant outgroups (the alpha, 


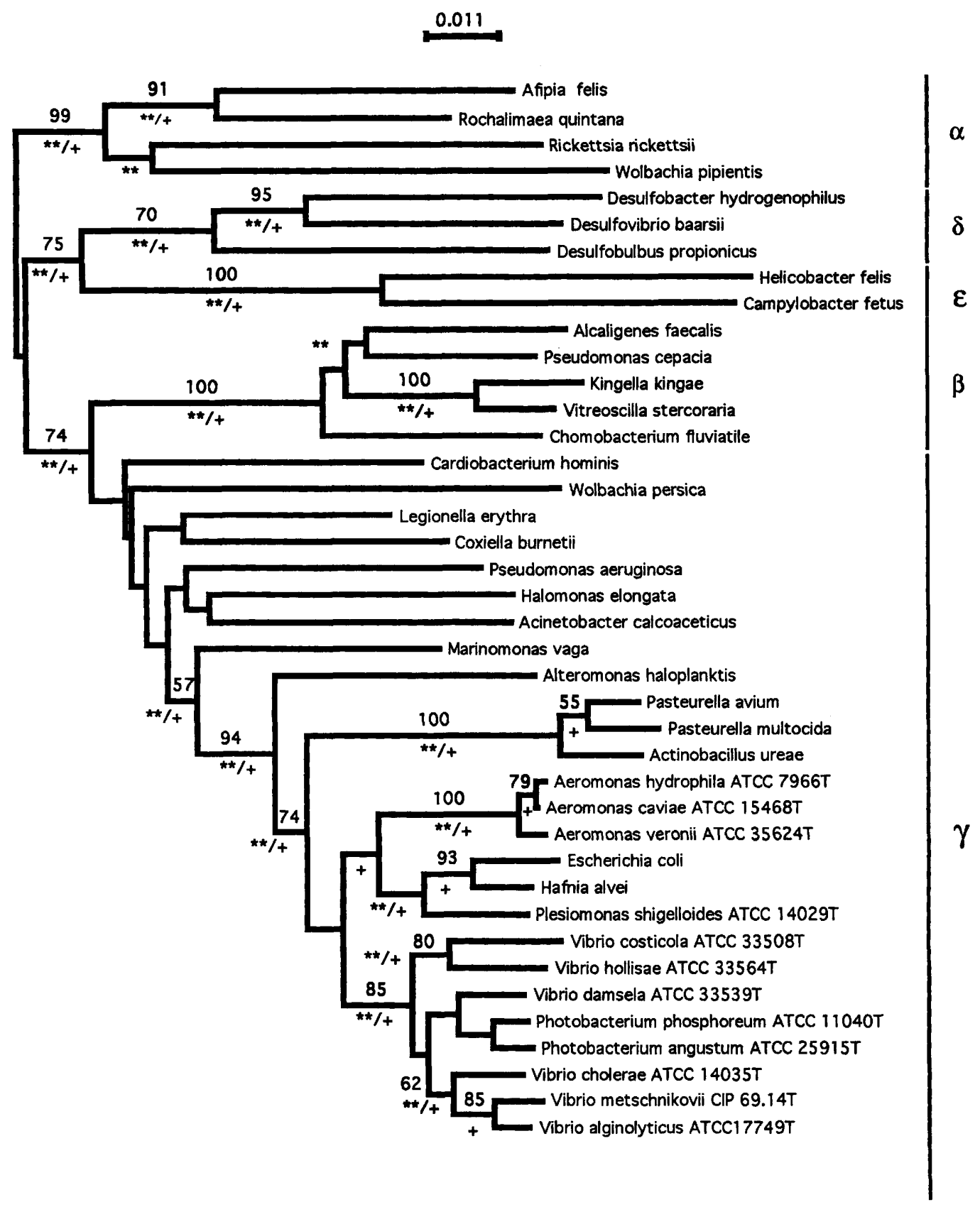

FIG. 1. Phylogenetic positions of the genera Vibrio, Photobacterium, Aeromonas, and Plesiomonas within the Proteobacteria. We constructed unrooted trees showing the topologies obtained when we used the neighbor-joining method (the tree shown), the maximum-parsimony method, and maximum-likelihood method. The most parsimonious tree had an identical topology. The double asterisks indicate branches that connect taxa that were also found to be related by the maximum-likelihood method $(P<0.01)$. Scale bar $=0.011$ accumulated change per 100 nucleotides. A bootstrap analysis in which parsimony was used to assess the robustness of the topology (heuristic search, 100 replications) gave the values (expressed as percentages) above the lines (only values greater than $50 \%$ are shown).

beta, delta, and epsilon subgroups) and also because the branching orders were not supported by bootstrap data and were different depending on the method of phylogenetic analysis used. Low bootstrap values can indicate a weakly supported relationship, but they can also result from peculiar rates of mutation in some species or from the inclusion of outgroups that are too distantly related compared with the phylogenetic relationship being investigated. Thus, detailed phylogenies were investigated by using a restricted set of outgroups (see Materials and Methods).

Relative phylogenetic positions of the genera Vibrio, Photobacterium, Aeromonas, and Plesiomonas within the gamma subgroup. Since we had too many sequences to include them all in a single phylogenetic analysis (particularly for maximumlikelihood or maximum-parsimony analyses), a large number of analyses were performed by using different representatives 


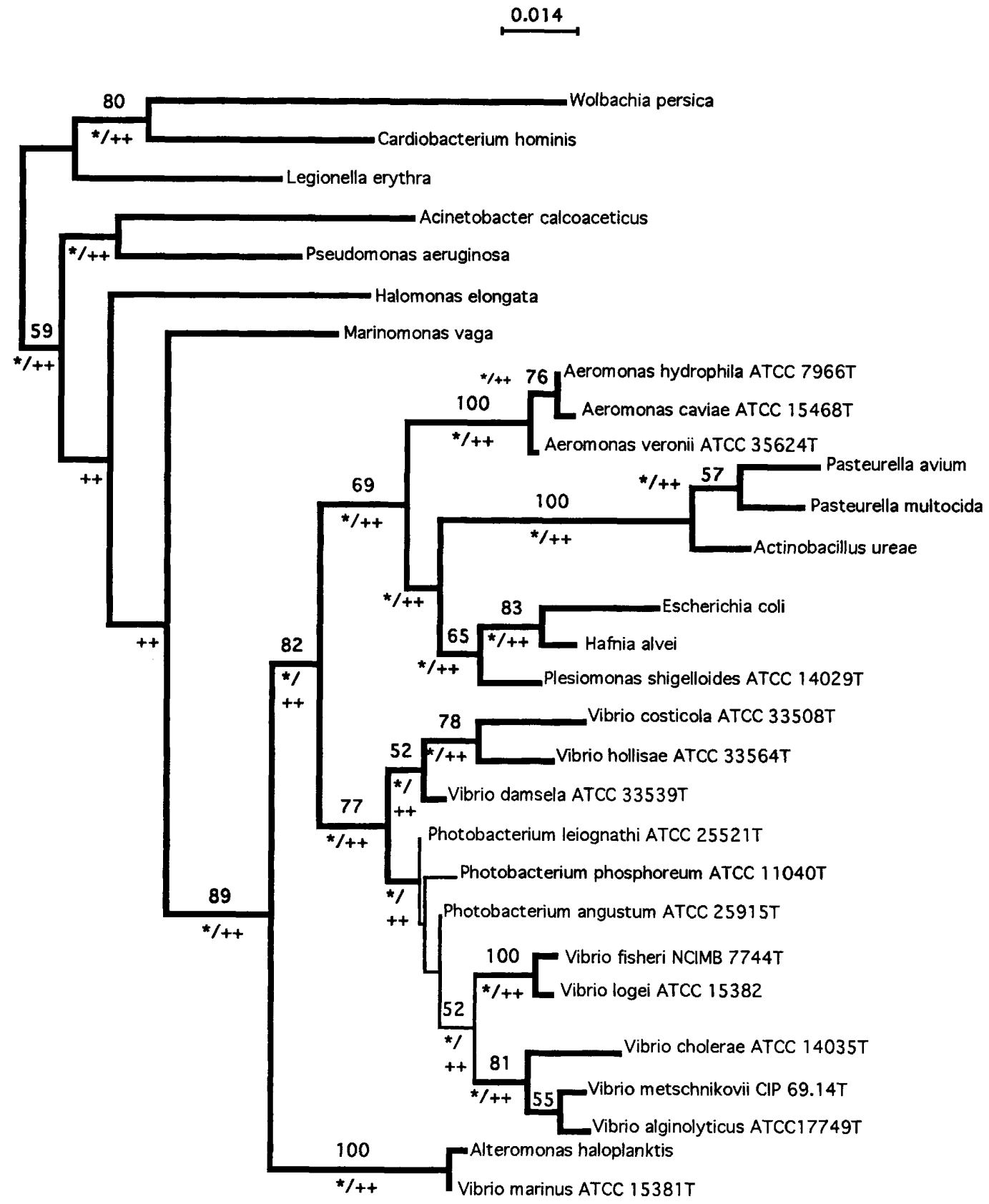

FIG. 2. Phylogenetic analysis restricted to the gamma subgroup of the Proteobacteria: unrooted tree obtained by the maximum-likelihood method. Double plus signs indicate that a branch is significantly positive $(P<0.01)$. Boldface lines indicate monophyletic taxa also found in the neighbor-joining analysis. The asterisks indicate taxa also found on the most-parsimonious tree. The numbers above the branches are the percentages determined in a bootstrap analysis. For additional explanations, see Materials and Methods.

of the four genera belonging to the Vibrionaceae or of the outgroups. All of the analyses gave similar results, and the results of two of the analyses are shown in Fig. 2 and 3.

Figure 2 is an unrooted tree that combines the results of a maximum-likelihood analysis (which are shown) with the results of neighbor-joining and maximum-parsimony analyses. A comparison of the different methods, as well as bootstrap replications, revealed a solid group consisting of the following four robust monophyletic units: the Aeromonadaceae, the Enterobacteriaceae plus the genus Plesiomonas, the Pasteurellaceae, and the genus Photobacterium plus the genus Vibrio.
The outgroups closest to these four taxa were Alteromonas haloplanktis and, to lesser extents, $M$. vaga and Halomonas elongata. Surprisingly, one of the Vibrio species, Vibrio marinus, clustered very tightly with Alteromonas haloplanktis (but see the Discussion). The Photobacterium-Vibrio group appeared to be a sister group of the families Pasteurellaceae, Aeromonadaceae, and Enterobacteriaceae. The exact relationships among the latter taxa were more difficult to determine and, in particular, were not well supported by bootstrap data in the presence of distant taxa (see Fig. 2) or changed when different outgroups were used. 


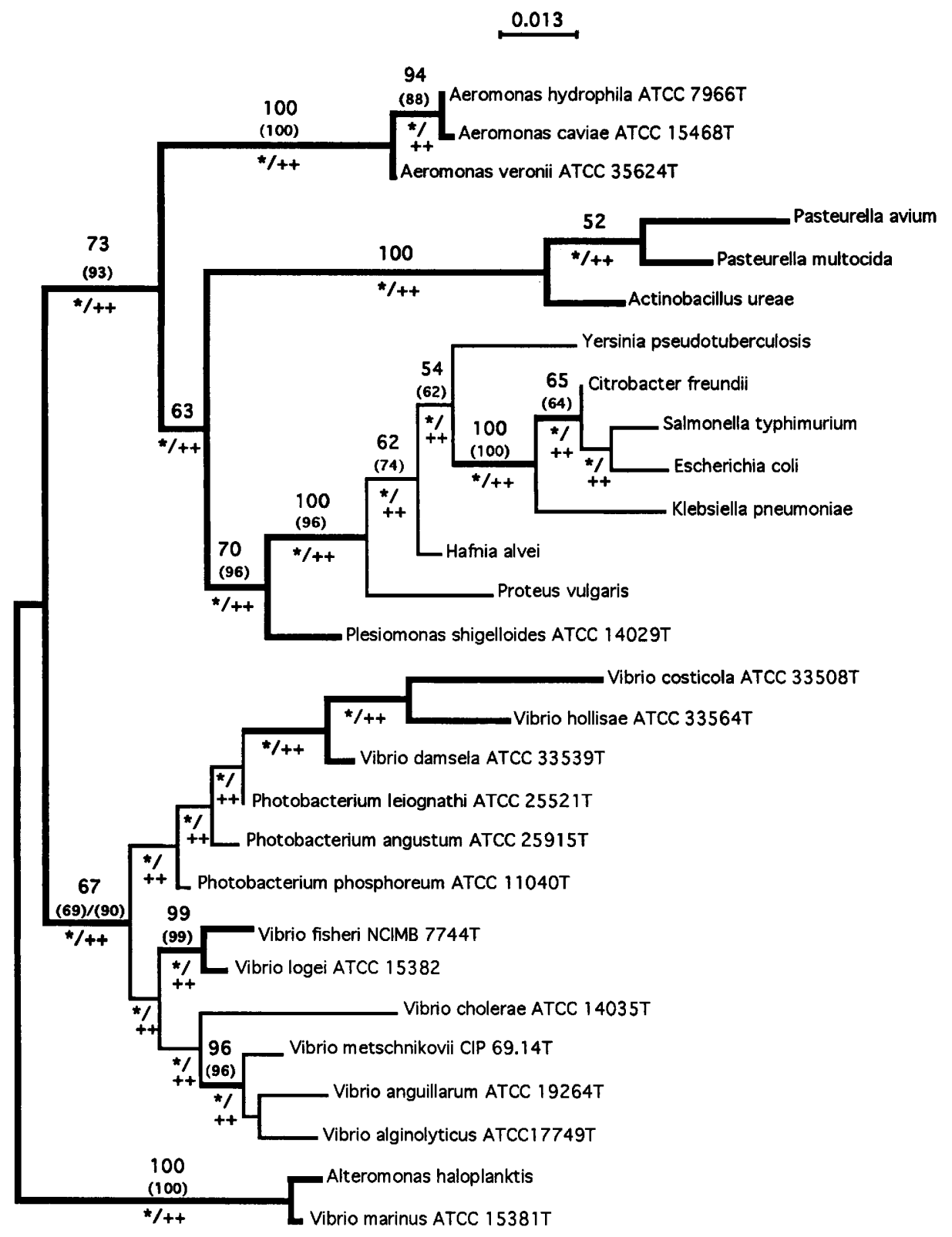

FIG. 3. Phylogenetic relationships among the Enterobacteriaceae, the Pasteurellaceae, and the genera Vibrio, Photobacterium, Aeromonas, and Plesiomonas. An unrooted tree was drawn as described in the legend to Fig. 2. The numbers in parentheses are the percentages of bootstrap replications when Pasteurella strains were omitted from the analysis. For the Vibrio-Photobacterium taxon, the number on the right is the value obtained when $V$. costicola was omitted from the analysis. For additional explanations, see Materials and Methods.

To resolve this problem, in another analysis we excluded the least closely related outgroups (Fig. 3). An association supported by the results of all three methods was then obtained between the Pasteurellaceae and the Enterobacteriaceae. In this analysis, some of the low bootstrap values may have resulted not from the presence of distant outgroups, but from the presence of species with unusually high rates of mutation (see Materials and Methods), such all of the members of the Pasteurellaceae. The deleterious effect of the presence of long branches is demonstrated in Fig. 3. When species on long branches were removed, (i) the removal of Vibrio costicola increased the bootstrap value from 69 to $90 \%$ for the branch uniting all species belonging to the genera Photobacterium and
Vibrio and (ii) the removal of the members of the Pasteurellaceae increased the bootstrap value from 70 to $96 \%$ for the branch uniting Plesiomonas shigelloides and the Enterobacteriaceae. In fact, removing the members of the Pasteurellaceae from the analysis increased the significance of almost all of the internal branches (numbers in parentheses in Fig. 3).

Intrageneric relationships among Vibrio and Photobacterium species. The relationships among all of the Vibrio and Photobacterium species for which the small-subunit rRNA sequences were known (Table 1) were investigated by all of the methods described above. The phylogenetic relationships of these species proved to be difficult to resolve (see Discussion), but three main groups were identified by all three methods and these 
results were reasonably well supported by bootstrap analysis data (data not shown). The three groups were (i) all Photobacterium species plus Vibrio damsela, $V$. costicola, and $V$. hollisae, (ii) Vibrio logei plus Vibrio fisheri, and (iii) all remaining Vibrio species listed in Table 1.

In addition, a number of pairs of taxa were clearly closely related (members of a monophyletic taxon as determined by all three methods). These pairs included Vibrio cholerae and Vibrio mimicus (100\% of bootstrap), Vibrio ordalii and $V$. anguillarum (100\%), Vibrio fluvialis and Vibrio furnisii (84\%), and finally, but not surprisingly, the two strains of Vibrio metschnikovii (73\%). $V$. costicola and $V$. hollisae were found to be related by all three methods (the relationship was supported by a moderate bootstrap value, $71 \%$ ), but their monophyly could have been an artifact caused by the well-known effect of long-branch attraction.

\section{DISCUSSION}

Comparisons of nearly complete small-subunit rRNA sequences confirmed that the genera Vibrio, Photobacterium, Aeromonas, and Plesiomonas are phylogenetically linked with the Enterobacteriaceae and Pasteurellaceae to form a monophyletic unit. Our data suggest that this topology is robust since it was obtained independently of the species selected as outgroups and of the phylogenetic method used. This result was also supported by very high bootstrap replication values (Fig. 1). Thus, these taxa form a monophyletic unit within the gamma 3 subgroup of the Proteobacteria (43). The genus Alteromonas appears to belong to this cluster, as a sister group, but the presence of a single representative sequence for all Alteromonas species precludes any definitive conclusion. We examined the relative positions of the families Vibrionaceae (restricted to the genera Vibrio and Photobacterium), Aeromonadaceae, Enterobacteriaceae, and Pasteurellaceae, and our data identified the Vibrionaceae as the deepest radiation and a sister group of the three other families (Fig. 2 and 3). The relative positions of the Aeromonadaceae, Enterobacteriaceae, and Pasteurellaceae are more difficult to determine, most probably because of the long internal branches without internodes that characterize the Aeromonadaceae and the Pasteurellaceae and also because bacteria belonging to the Pasteurellaceae (13) seem to have a high rate of mutation. Previous reports based on small-subunit rRNA oligonucleotide cataloging (18), rRNA-DNA hybridization $(6,7,18)$, 5S rRNA sequencing $(12,28)$, and analyses of small-subunit rRNA sequences $(23,29,30)$ readily identified the genus Aeromonas as a well-defined monophyletic taxon but were contradictory with regard to the phylogenetic position of this taxon. This is not surprising since the genus Aeromonas is characterized by a long internal branch and its position can be artifactual in some analyses, making it appear to be a radiation deeper than it should be; we have observed that neighbor-joining methods are sometimes more sensitive to such problems, probably because they work by grouping close neighbors first. Our results that strongly support the hypothesis that the Aeromonadaceae is a sister group of the Enterobacteriaceae and Pasteurellaceae were derived from longer sequences with more constant domains and/or more sequences than the results of previous studies, and the validity of the different topologies was assessed by using several methods and bootstrap analysis. In conclusion, although they are clearly genetically related, the four genera previously placed in the family Vibrionaceae can no longer be placed in a single taxon on the basis of genetic data and, more specifically, small-subunit rRNA sequence data (see below).
Our analyses revealed a robust monophyletic unit formed by Plesiomonas shigelloides and the Enterobacteriaceae (Fig. 3), which is in agreement with 5S rRNA data that also relate Plesiomonas shigelloides to the genus Proteus (27) and with previous small-subunit rRNA analysis data (29). Our data show that Plesiomonas shigelloides is genetically more closely related to Proteus vulgaris than to any other bacterium represented in the set of sequences available (Fig. 3). Plesiomonas shigelloides branched deeply, as a sister group of all of the other enterobacteria sequenced; more data are necessary to decide whether a new family, the Plesiomonadaceae, with one species, Plesiomonas shigelloides, should be created or whether this species should be placed in the Enterobacteriaceae.

The rather long internal branch and deep radiation that isolate all of the Aeromonas species sequenced from all other bacteria (Fig. 3) support the decision of the International Committee on Systematic Bacteriology Subcommittee on the Taxonomy of Vibrionaceae (21) to accept the new family Aeromonadaceae (as proposed by Colwell et al. [12]) to comprise the single genus Aeromonas. This long internal branch suggests that all known (sequenced) species in this genus might be derived from a recent common ancestor. It is difficult (i) to determine a root for the Aeromonadaceae (because there is no very closely related outgroup), and (ii) to determine with precision the internal phylogeny of the Aeromonadaceae (there are few differences between species). Although these circumstances could in part explain the disagreement observed previously between the results of small-subunit rRNA analysis and the results of chromosomal DNA-DNA pairing experiments (29), recently Sneath (36) also suggested that recombination events between rRNA genes might significantly affect all phylogenetic methods in which small-subunit rRNA sequences are used to determine relationships between Aeromonas species.

A restricted family Vibrionaceae that includes two genera, Vibrio and Photobacterium, was supported by the results of all of the methods used (Fig. 2 and 3). Bootstrap values increased when species such as $V$. cholerae or $V$. costicola were removed, a result that we interpret more as an artifact resulting from the high mutation rate in the rRNA sequences of $V$. cholerae or $V$. costicola than an indication that these bacteria do not belong to the Vibrionaceae. Thus, most Vibrio and Photobacterium species form a robust monophyletic unit; the single exception is $V$. marinus (Fig. 2 and 3 ). The external position of $V$. marinus has also been observed during analyses of 5S rRNA sequences (26, 27) and partial small-subunit rRNA sequences (23). Our Alteromonas haloplanktis sequence suggests a close link with $V$. marinus. However, several differences between the corresponding parts of our complete $V$. marinus sequence and the partial sequences published previously (23) are difficult to explain by a difference in strains. It is likely that one of these sequences is not "true" $V$. marinus sequence, and the exact phylogenetic position of $V$. marinus remains to be determined. Within the restricted family Vibrionaceae, our only robust result was a division of all species into three subgroups. Other relationships proved to be extremely difficult to establish with certainty, probably for two reasons: (i) the members of some branches exhibited particularly high rates of mutation (typically $V$. costicola and $V$. hollisae), while the members of other branches exhibited low rates of mutation (for example, $V$. proteolyticus and $V$. logei) and (ii) many species exhibited low numbers of differences in their rRNA sequences (Table 2 shows distances between sequences). While some of these problems could possibly be tackled by using a more sophisticated approach than the methods that we employed, the low numbers of differences between many pairs of sequences and 


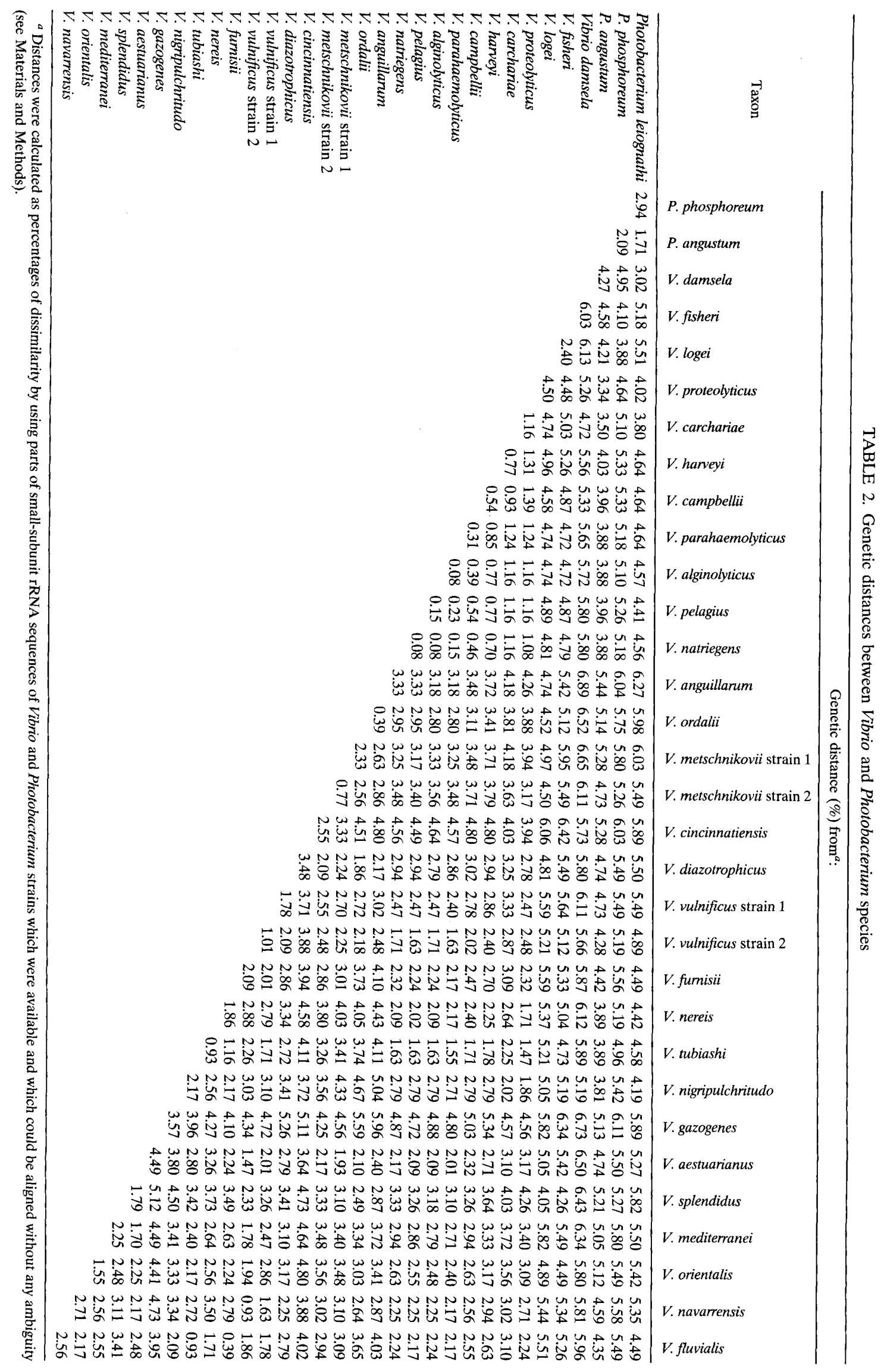


the possible existence of crossing-over (as observed for Aeromonas small-subunit sequences [36]) suggest that sequences from a different part of the genome will probably be required to help answer these questions.

Within the restricted family Vibrionaceae, it would certainly be interesting to compare the results obtained from a sequence analysis with data derived from numerical taxonomy. However, this task proved to be very difficult, because relatively few classical studies have included all species, and because some strains have sometimes been wrongly recognized as species (see below). Importantly, in none of the classical numerical taxonomy studies did the authors examine their proposed taxonomic schemes for robustness, and it is very difficult to assess which part of an interpretation relied on extremely solid data and which part was more an extrapolation. The results of a recent thorough study (19) in which the authors used numerical taxonomy (61 different phenotypic tests) to examine 236 strains belonging to the Vibrionaceae (extended definition) also suggest that there may be an additional problem. An ordination analysis did not provide a clear separation of vibrios, enteric bacteria, and Aeromonas species, in contrast to what is now generally admitted and, in particular, to what had been found by workers in the same laboratory on the basis of an analysis of $5 \mathrm{~S}$ rRNA sequences (28). Grimes et al. suggested that the phenotypes of species freshly isolated from natural environments could be different from the phenotypes of the same species after some period of laboratory culture. As part of their phenotypes, bacteria certainly contain the products of many regulated genes that are expressed according to the environment in which they grew. Thus, environmentally-linked phenotypes could be reflections of the ecosystems from which the bacteria were obtained, and they could occur for more than several cell cycles (a phenomenon analogous to magnetic remanence). If this phenomenon does exist, as suggested by a comparison of $V$. parahaemolyticus strains isolated from human and marine sources (3), analysis of small-subunit rRNA (or some other structural gene) is certainly a better phylogenetic index and a more efficient taxonomic tool, but phenotypic analysis is the only method that can be used to determine the actual place of a particular microorganism in a natural food web. However, because possible errors in determining sequences could lead to misidentification of species, and because it is not yet known how to relate exactly classical strain identification to small-subunit rRNA sequences, we concluded that it would be wise to reinvestigate previously published molecular data and/or strain identification, at least, when molecular phylogenies (often derived from only a single strain) are in strong disagreement with the results of classical taxonomy derived from repeated biochemical studies. The vibrios are a particularly difficult problem, because a few isolates have probably been misidentified in some studies and because of the phenotypic variations mentioned above (for an example of discrepancy, see references 3 and 11).

For phylogeny and perhaps for sound taxonomy, comparison of sequences is an appropriate approach only if there has been no lateral transfer of genes and if most (or all) bacterial species follow a vertical scheme of evolution. Baumann et al. have analyzed the evolution of rRNA (6), alkaline phosphatase, glutamine synthetase, and superoxide dismutase $(4,5)$ in Vibrio and Photobacterium species (8). The high correlation coefficients for the evolutionary pathways of these different molecules led these authors to assume that there has been no major lateral transfer of genes. Under these conditions, the phylogenetic reconstruction obtained from one molecule (a gene tree) represents the evolutionary history of the species (a species tree). Since rRNA sequences are now obtained more easily than the sequence of any other gene, we rely on such sequences for reconstructing phylogenies from sequences and for estimating tree robustness. However, small-subunit rRNA analysis has some limitations: (i) small-subunit rRNA sequences do not contain highly variable domains that are probably required to properly analyze relationships of closely related organisms (similar species or strains within species), (ii) crossing over between genes of a multigene family (such as rRNA) could affect the reconstruction of phylogenies for closely related species (36), and (iii) bacterial classification should not be restricted to the evolutionary history of a single gene, since the definition of a species is related at least as much to its role in an ecosystem as to its ribosomal history. It is now important to try to correlate the information derived from rRNA analyses with the considerable amount of phenotypic analysis and numerical taxonomy data available $(10,24,39)$, bearing in mind that some or many of the phenotypic traits usually analyzed might be short-term adaptative characteristics expressed by a "species" to survive in a particular environment.

\section{ACKNOWLEDGMENTS}

This work was supported by INSERM and CNRS and by a fellowship from the Fondation pour la Recherche Médicale and the M.R.E. to R.R.

We thank two anonymous referees for their detailed reviews and very helpful comments.

\section{REFERENCES}

1. American Type Culture Collection. 1992. American Type Culture Collection catalogue of bacteria and bacteriophages, 18th ed. American Type Culture Collection, Md.

2. Anderson, R. D., C.-Y. Bao, D. T. Minnick, M. Veigl, and W. D. Sedwick. 1992. Optimization of double-stranded DNA sequencing for polymerase chain reaction products, p. 39-40 and 57-58. In Editorial comments, vol. 19. United States Biochemical Corp., Cleveland.

3. Anderson, R. S., and E. J. Ordal. 1975. Deoxyribonucleic acid relationships among marine vibrios. J. Bacteriol. 109:696-706.

4. Bang, S. S., L. Baumann, J. Woolkalis, and P. Baumann. 1981. Evolutionary relationships in Vibrio and Photobacterium as determined by immunological studies of superoxide dismutase. Arch. Microbiol. 130:111-120.

5. Baumann, L., S. S. Bang, and P. Baumann. 1980. Study of relationship among species of Vibrio, Photobacterium, and terrestrial enterobacteria by an immunological comparison of glutamine synthetase and superoxide dismutase. Curr. Microbiol. 4:133-138.

6. Baumann, L., and P. Baumann. 1976. Study of the relationships among marine and terrestrial enterobacteria by means of in vivo DNA/ribosomal RNA hybridization. Microbios Lett. 3:11-20.

7. Baumann, P., and L. Baumann. 1981. The marine gram-negative eubacteria: genera Photobacterium, Beneckea, Alteromonas, Pseudomonas, and Alcaligenes, p. 1352-1394. In M. P. Starr, H. Stolp, H. G. Trüper, A. Balows, and H. G. Schlegel (ed.), The prokaryotes. A handbook on habitats, isolation and identification of bacteria. Springer Verlag, New York.

8. Baumann, P., L. Baumann, M. J. Woolkalis, and S. S. Bang. 1983. Evolutionary relationships in Vibrio and Photobacterium: a basis for a natural classification. Annu. Rev. Microbiol. 37:369-398.

9. Baumann, P., and R. H. W. Schubert. 1984. Family II. Vibrionaceae, p. 516-517. In N. R. Krieg and J. G. Holt (ed.), Bergey's manual of systematic bacteriology, vol. 1. The Williams and Wilkins Co., Baltimore.

10. Boe, B., and J. Gjerde. 1980. Fatty acid patterns in the classification of some representatives of the families Enterobacteriaceae and Vibrionaceae. J. Gen. Microbiol. 116:41-49.

11. Citarella, R. V., and R. R. Colwell. 1970. Polyphasic taxonomy of the genus Vibrio: polynucleotide sequence relationships among selected Vibrio species. J. Bacteriol. 104:434-442.

12. Colwell, R. R., M. T. MacDonell, and J. De Ley. 1986. Proposal to recognize the family Aeromonadaceae fam. nov. Int. J. Syst. 
Bacteriol. 36:473-477.

13. Dewhirst, F. E., B. J. Paster, I. Olsen, and G. J. Fraser. 1992. Phylogeny of 54 representative strains of species in the family Pasteurellaceae as determined by comparison of 16S rRNA sequences. J. Bacteriol. 174:2002-2013.

14. Dorsch, M., D. Lane, and E. Stackebrandt. 1992. Towards a phylogeny of the genus Vibrio based on $16 \mathrm{~S}$ rRNA sequences. Int. J. Syst. Bacteriol. 42:58-63.

15. Erdmann, V. A., J. Wolters, E. Huysman, and R. De Wachter. 1985. Collection of published 5S, 5.8S and 4.5S ribosomal RNA sequences. Nucleic Acids Res. 13:105-153.

16. Farmer, J. J., III. 1991 . The family Vibrionaceae, p. 2938-2951. In A. Balows, H. G. Trüper, M. Dworkin, W. Harder, and K.-H. Schleifer (ed.), The prokaryotes. A handbook on the biology of bacteria: ecophysiology, isolation, identification, applications. Springer-Verlag, New York.

17. Felsenstein, J. 1990. PHYLIP manual, version 3.3. University Herbarium, University of California, Berkeley.

18. Fox, G. E., E. Stackebrandt, R. B. Hespell, J. Gibson, J. Maniloff, T. A. Dyer, R. S. Wolfe, W. E. Balch, R. S. Tanner, L. J. Magrum, L. B. Zablen, R. Blakemore, R. Gupta, L. Bonen, B. J. Lewis, D. A. Stahl, K. R. Luehrsen, K. N. Chen, and C. R. Woese. 1980. The phylogeny of prokaryotes. Science. 209:457-463.

19. Grimes, D. J., D. Jacobs, P. R. Swartz, P. R. Brayton, and R. R. Colwell. 1993. Numerical taxonomy of gram-negative, oxidasepositive rods from carcharhinid sharks. Int. J. Syst. Bacteriol. 43:88-98.

20. Huelsenbeck, J. P., and D. M. Hillis. 1993. Success of phylogenetic methods in the four-taxon case. Syst. Biol. 42:247-264.

21. International Committee on Systematic Bacteriology Subcommittee on the Taxonomy of Vibrionaceae. 1992. Minutes of the meeting. Int. J. Syst. Bacteriol. 42:199-201.

22. Kim, J. 1993. Improving the accuracy of phylogenetic estimation by combining different methods. Syst. Biol. 42:331-340.

23. Kita-Tsukamoto, K., H. Oyaizu, K. Nanba, and U. Simidu. 1993. Phylogenetic relationships of marine bacteria, mainly members of the family Vibrionaceae, determined on the basis of 16S rRNA sequences. Int. J. Syst. Bacteriol. 43:8-19.

24. Lambert, M. A., F. W. Hickman-Brenner, J. J. Farmer III, and C. W. Moss. 1983. Differentiation of Vibrionaceae species by their cellular fatty acid composition. Int. J. Syst. Bacteriol. 33:777-792.

25. Lane, D. J., B. Pace, G. J. Olson, D. A. Stahl, M. L. Sogin, and R. Pace. 1985. Rapid determination of $16 \mathrm{~S}$ ribosomal RNA sequences for phylogenetic analyses. Proc. Natl. Acad. Sci. USA 82:6955-6959.

26. MacDonell, M. T., and R. R. Colwell. 1984. Nucleotide base sequence of Vibrionaceae 5S rRNA. FEBS Lett. 175:183-188.

27. MacDonell, M. T., and R. R. Colwell. 1985. Phylogeny of the Vibrionaceae and recommendations for two new genera, Listonella and Shewanella. Syst. Appl. Microbiol. 6:171-182.

28. MacDonell, M. T., D. G. Swartz, B. A. Ortiz-Conde, G. A. Last, and R. R. Colwell. 1986. Ribosomal RNA phylogenetics for the vibrio-enteric group of eubacteria. Microbiol. Sci. 3:172-179.

29. Martinez-Murcia, A. J., S. Benlloch, and M. D. Collins. 1992. Phylogenetic interrelationships of members of the genera Aeromonas and Plesiomonas as determined by $16 \mathrm{~S}$ ribosomal DNA sequencing: lack of congruence with results of DNA-DNA hybridations. Int. J. Syst. Bacteriol. 42:412-421.

30. Martinez-Murcia, A. J., C. Esteve, E. Garay, and M. D. Collins. 1992. Aeromonas allosaccharophila sp. nov., a new mesophilic member of the genus Aeromonas. FEMS Microbiol. Lett. 91:199206.

31. Qu, L. H., B. Michot, and J.-P. Bachellerie. 1983. Improved methods for structure probing in large RNAs: a rapid "heterologous" sequencing approach is coupled to the direct mapping of nuclease accessible sites. Nucleic Acids Res. 11:5903-5920.

32. Reichelt, J. L., P. Baumann, and L. Baumann. 1976. Study of genetic relationships among marine species of the genera $B e$ neckea and Photobacterium by means of in vitro DNA/DNA hybridization. Arch. Microbiol. 110:101-120.

33. Saitou, N., and M. Nei. 1987. The neighbor-joining method: a new method for reconstructing phylogenetic trees. Mol. Biol. Evol. 4:406-425.

34. Sanger, F., S. Nicklen, and A. R. Coulson. 1977. DNA sequencing with chain-terminating inhibitors. Proc. Natl. Acad. Sci. USA 74:967-973.

35. Smith, A. B., B. Lafay, and R. Christen. 1992. Comparative variation of morphological and molecular evolution through geologic time: 28S ribosomal RNA versus morphology in echinoids. Philos. Trans. R. Soc. Lond. Biol. Sci. 338:365-382.

36. Sneath, P. H. A. 1993. Evidence from Aeromonas for genetic crossing-over in ribosomal sequences. Int. J. Syst. Bacteriol. 43:626-629.

37. Stackebrandt, E., and C. R. Woese. 1984. The phylogeny of prokaryotes. Microbiol. Sci. 1:117-122.

38. Swofford, D. 1990. PAUP: phylogenetic analysis using parsimony, version 3.0. Natural History Survey, Champaign.

39. Urdacci, M. C., M. Marchand, and P. A. D. Grimont. 1990. Characterization of 22 Vibrio species by gas chromatography analysis of their cellular fatty acids. Res. Microbiol. 141:437-452.

40. Valle, O., M. Dorsh, R. Wijk, and E. Stackebrandt. 1990. Nucleotide sequence of the 16S rRNA from Vibrio anguillarum. Syst. Appl. Microbiol. 13:257.

41. Véron, M. 1966. Taxonomie numérique des vibrions et de certaines bactéries comparables. Ann. Inst. Pasteur (Paris) 111:671-709.

42. Véron, M. M. 1965. La position taxonomique des Vibrio et de certaines bactéries comparables. C. R. Acad. Sci. 261:5243-5246.

43. Woese, C. R., W. G. Weisburg, C. M. Hahn, B. J. Paster, L. B. Zablen, B. J. Lewis, T. J. Macke, W. Ludwig, and E. Stackebrandt. 1985. The phylogeny of purple bacteria: the gamma subdivision. Syst. Appl. Microbiol. 6:25-33. 